\title{
Tetragonally Packed Cylinder Structure via Hierarchical Assembly of Comb-Coil Diblock Copolymer
}

\author{
Hsin-Lung Chen, ${ }^{*} \dagger$ Jiang-Shing Lu, ${ }^{\dagger}$ Chi-Heisn Yu, ${ }^{\dagger}$ Chao-Lin Yeh, ${ }^{\dagger}$ U-Ser Jeng,,${ }^{\dagger}$ and \\ Wen-Chang Chen ${ }^{\S}$
}

\begin{abstract}
Department of Chemical Engineering, National Tsing Hua University, Hsin-Chu 30013, Taiwan, National Synchrotron Radiation Research Center, Hsin-Chu 300, Taiwan, and Department of Chemical Engineering and Institute of Polymer Science and Engineering, National Taiwan University,
\end{abstract} Taipei 10617, Taiwan

Received November 14, 2006; Revised Manuscript Received February 19, 2007

\begin{abstract}
Block copolymers constitute an important class of soft material capable of self-assembling into nanoscale microdomains with well-defined geometries. The cylindrical domains thus formed have been found to pack almost exclusively in hexagonal lattice. In this study, we disclose how the mesomorphic molecular order can mediate the packing symmetry to generate an anomalous tetragonal lattice in a diblock copolymer composing of a coil and a supramolecular comb block. This system displays a hierarchical structure in which the largerscale cylinders formed by the coil blocks are embedded in a liquid crystalline matrix composing of a smallerscale lamellar structure organized by the comb blocks. The cylinders are found to pack in either the typical hexagonal lattice or the tetragonal lattice governed by the orientation of the lamellae with respect to the cylinder prescribed by the overall grafting density of the comb blocks.
\end{abstract}

\section{Introduction}

Self-assembly of soft matter is one of the most effective approaches for constructing nanostructures with controllable order. Many of these structures are mesomorphic, characterized by certain degrees of orientational or positional order at one or multiple length scales coupled with the dynamic spectrum over a broad range of time scale. ${ }^{1}$ Soft matter has been considered as a key element for producing the newest generation of smart and functional materials; therefore, the development of the fundamental basis for engineering its self-assembly behavior is an essential step toward nanoscale regulation of structure and functioning.

Block copolymers composing of two or more covalently connected chains per molecule constitute an important class of soft material capable of self-assembling into a rich variety of nanostructures. ${ }^{2,3}$ Two levels of structural organization can be recognized for block copolymer self-assembly in the melt state. The first level is the microphase separation between the repulsive dissimilar blocks, which generates micelle composing of the microdomain (core) and the corona formed by the respective blocks. The geometry of the microdomain thus formed includes lamellae, cylinders, and spheres depending on the interfacial tension at domain interface and the conformational entropy of the block chains. ${ }^{3,4}$ The second level involves the spatial organization of the micelles, which results in long-range ordered packing of the microdomains with the lattice symmetry governed by the domain geometry and the degree of deformation of the micelle corona for space filling. The lattice with minimum corona deformation is normally favored. ${ }^{4}$

The cylindrical microdomains formed by block copolymers have been found to organize almost exclusively in a hexagonal lattice. Hexagonal packing is indeed the predominant structure among the materials composing of cylindrical supramolecular

\footnotetext{
Department of Chemical Engineering, National Tsing Hua University.

$\doteqdot$ National Synchrotron Radiation Research Center.

$\S$ Department of Chemical Engineering and Institute of Polymer Science and Engineering, National Taiwan University.
}

building blocks. Tetragonal lattice is another type of packing symmetry which may be observed under the regulation of some special interactions. Examples of tetragonal systems include T-shaped liquid crystals with the nonpolar lateral side chains forming cylindrical domains, ${ }^{5,6}$ the complexes of cationic dendronized polymers with anionic lipids with the polymer backbone representing the cylinder, ${ }^{7}$ rod-coil multiblock liquid crystalline oligomers with the rods forming the cylinders ${ }^{8}$ and DNA/dendrimer complexes with the DNA rods organizing into tetragonal lattice for effective charge matching. ${ }^{9,10}$ The characteristic lengths of the nanostructures in these systems are of several nanometers. Tetragonal lattices with the length scale of several tens of $\mathrm{nm}$ have been observed for linear and heteroarm ABC triblock copolymers composing of two types of cylinders formed by two of the block chains. ${ }^{11,12}$ For diblock copolymers containing only one type of cylinder, tetragonal lattice has been reported for polystyrene-block-poly(2-(3cholesteryl-oxycarbonyloxy)ethyl methacrylate) (PS- $b$-PChEMA), in which the PS cylinders were embedded within a liquid crystalline matrix formed by PChEMA block possessing cholesteryl mesogen. ${ }^{13}$ Though not a cylinder-forming system, a recent study has shown that a rod-coil block copolymer with a mesogen jacketed liquid crystalline block exhibited a tetragonal perforated layer structure as opposed to the hexagonal perforation in the coil-coil systems. ${ }^{14}$

In the present study, we reveal that a single diblock copolymer system with comb-coil molecular architecture can exhibit both hexagonally and tetragonally packed cylinder structures. This comb-coil architecture is constructed by a supramolecular approach first demonstrated by Ikkala et al. ${ }^{15-22}$ through selective complexation of an amphiphilic surfactant, dodecylbenzenesulfonic acid (DBSA), with the poly(4-vinylpyridine) (P4VP) block in a coil-coil polystyrene-block-poly(4-vinylpyridine) (PS- $b$-P4VP), as schematically illustrated in Figure 1. The resultant comb block, denoted by P4VP(DBSA), consists of the DBSA side chains electrostatically bound to the P4VP backbone. Two types of repulsive interaction existing in this 

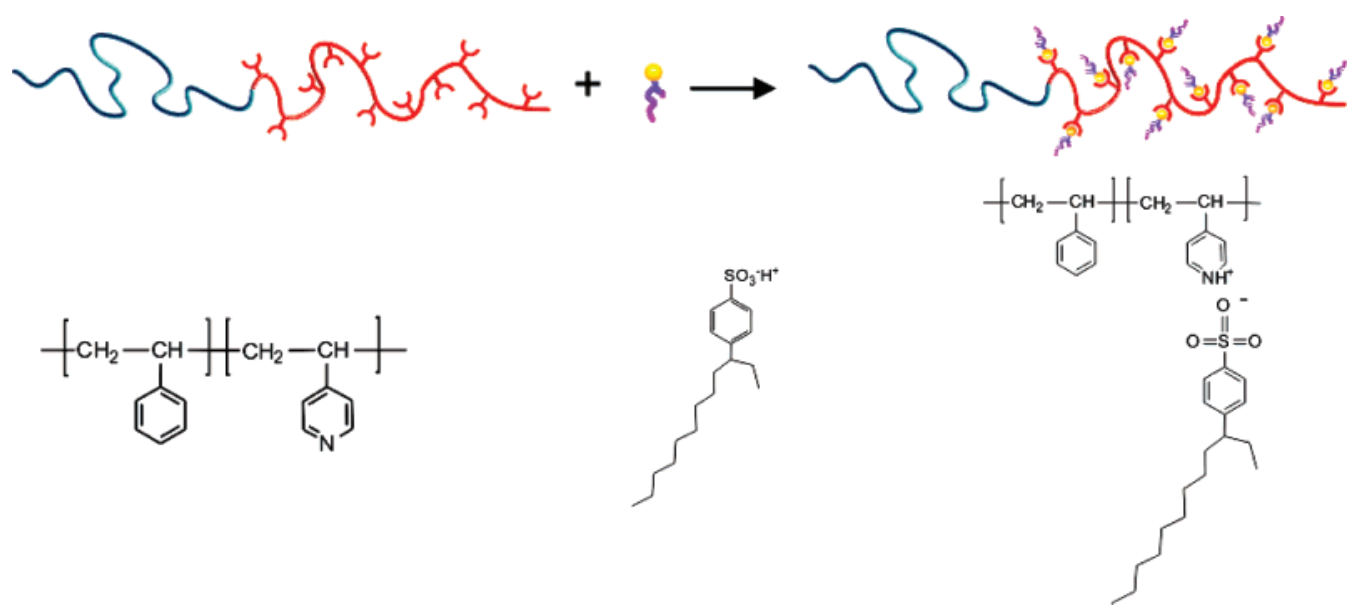

Figure 1. Schematic illustration of the preparation of the comb-coil diblock copolymer through selective complexation of an amphiphilic surfactant, DBSA, with the P4VP block in a coil-coil PS- $b$-P4VP.

Table 1. Overall Binding Fractions $(x)$ and the Corresponding Volume Fractions of PS Block $\left(f_{\mathrm{PS}}\right)$ of the Two PS- $b$-P4VP(DBSA) Comb-Coil Copolymer Systems under Study

\begin{tabular}{clll}
\hline & $x$ & \multicolumn{1}{c}{$f_{\mathrm{PS}}$} & \multicolumn{1}{c}{ morphology $^{a}$} \\
\hline PS12K- $b$-P4VP12K & 0.0 & 0.53 & lamellae \\
$M_{\mathrm{b}, \mathrm{PS}}=12000$ & 0.5 & 0.28 & TETRA cylinder-within-lamellae \\
$M_{\mathrm{b}, \mathrm{P} 4 \mathrm{VP}}=11800$ & 0.6 & 0.26 & TETRA cylinder-within-lamellae \\
$M_{\mathrm{w}} / M_{\mathrm{n}}=1.05$ & 0.75 & 0.23 & HEX cylinder-within-lamellae \\
& 1.0 & 0.19 & HEX cylinder-within-lamellae \\
& 0.0 & 0.52 & lamellae \\
PS50K- $b$-P4VP50K & 0.0 . \\
$M_{\mathrm{b}, \mathrm{PS}}=50$ 000; & 0.5 & 0.28 & TETRA cylinder-within-lamellae \\
$M_{\mathrm{b}, \mathrm{P} 4 \mathrm{VP}}=49$ 800; & 0.6 & 0.26 & TETRA cylinder-within-lamellae \\
$M_{\mathrm{w}} / M_{\mathrm{n}}=1.05$ & 0.75 & 0.23 & HEX cylinder-within-lamellae \\
& 1.0 & 0.19 & HEX cylinder-within-lamellae
\end{tabular}

${ }^{a}$ TETRA and HEX stand for tetragonal and hexagonal lattice, respectively.

comb-coil copolymer lead to microphase separations at two distinct length scales. ${ }^{15,16,21}$ The repulsion between PS coil and P4VP(DBSA) comb generates the microdomains composing of the respective blocks with the length scale of tens of $\mathrm{nm}$. In the domains containing P4VP(DBSA) blocks, a lamellar mesophase with the length scale of several nanometers forms due to the microphase separation between the polar backbone and the nonpolar side chains in the comb blocks. Here we have properly chosen the molecular weight of PS- $b$-P4VP and the overall binding fraction of DBSA to P4VP block, such that the system exhibits cylinder-within-lamellae morphology, in which the PS cylindrical domains are embedded in the matrix composing of P4VP(DBSA) lamellar mesophase. We will show that the PS cylinders arrange in the typical hexagonal lattice or an anomalous tetragonal lattice depending on the overall binding fraction. The present system is uniquely different from PS- $b$-PChEMA ${ }^{13}$ in terms of the tunable packing symmetry and the special molecular architecture constructed by a simple supramolecular approach.

\section{Experimental Section}

Materials. Two symmetric PS- $b$-P4VPs, denoted as PS12K- $b$ P4VP12K and PS50K- $b$-P4VP50K and synthesized by sequential anionic polymerizations of styrene and 4-vinylpyridine, were acquired from Polymer Source, Inc. The number-average molecular weights of PS and P4VP blocks in the former are 12000 and 11800, respectively, whereas those in the later are 50000 and 49800, respectively. The polydispersity indices of both samples are 1.05. The surfactant DBSA (soft type) was obtained from Tokio Kasei, Tokyo, Japan. This material has the purity of $90 \%$, and the remaining impurity consists of different chain lengths and branches: $\left(\mathrm{C}_{n} \mathrm{H}_{2 n+1}\right)\left(\mathrm{C}_{m} \mathrm{H}_{2 m+1}\right) \mathrm{CHPhSO}_{3} \mathrm{H}$, with $n+m+1=10$, ..., 14. It was dried in vacuo at $60{ }^{\circ} \mathrm{C}$ for $12 \mathrm{~h}$ before use.

Preparation of Comb-Coil Diblock Copolymers. PS- $b$-P4VP(DBSA) comb-coil copolymers were prepared by dissolving preweighed PS- $b$-P4VP and DBSA in $N, N$-dimethylformamide (DMF) followed by evaporating most solvent at ca. $60{ }^{\circ} \mathrm{C}$. The samples were finally dried in vacuo at $100{ }^{\circ} \mathrm{C}$ for $12 \mathrm{~h}$ to remove the residual solvent. The overall binding fraction of DBSA to P4VP block is denoted by $x$, which expresses the average number of DBSA molecules bound with a 4VP monomer unit prescribed by the feed ratio of DBSA and P4VP block in the copolymers. The comb-coil copolymers with $x=0.5,0.6,0.75$, and 1.0 are prepared here, as they all display cylinder-within-lamellae morphology. The volume fraction of PS block ranges from 0.19 to 0.28 depending on $x$ (cf. Table 1). For the SAXS and TEM experiments, the samples were further annealed at $150{ }^{\circ} \mathrm{C}$ in vacuum for $30 \mathrm{~h}$ prior to the measurements.

Large-Amplitude Oscillatory Shear Experiment. Largeamplitude oscillating shear was performed to produce large-scale alignment of the microdomains in the samples with $x=1.0$ and 0.5 . The shear was carried out with a Linkam CSS 450 shear hot stage in the oscillatory mode with the shear frequency of $0.1 \mathrm{~Hz}$ and the strain amplitude of $50 \%$. The sample was first sheared at $135{ }^{\circ} \mathrm{C}$ for $1 \mathrm{~h}$ followed by cooling to $35^{\circ} \mathrm{C}$ at $2{ }^{\circ} \mathrm{C} / \mathrm{min}$.

Small-Angle X-ray Scattering (SAXS) Measurements. SAXS measurements were performed using a Bruker Nanostar SAXS instrument. The X-ray source, a 1.5 KW X-ray generator (Kristalloflex 760) equipped with a $\mathrm{Cu}$ tube, was operated at $35 \mathrm{~mA}$ and $40 \mathrm{kV}$. The scattering intensities and patterns were detected by a two-dimensional position-sensitive detector (Bruker AXS) with 512 $\times 512$ channels. The area scattering patterns of the isotropic samples have been radially averaged to generate the intensity profiles output as the plots of the scattering intensity $(I)$ vs the scattering vector, $q=4 \pi / \lambda[\sin (\theta / 2)](\theta=$ scattering angle $)$. The scattering intensities have been corrected by the empty beam scattering and the sensitivity of each pixel of the area detector.

Transmission Electron Microscopy (TEM) Experiment. The real-space morphology of PS- $b$-P4VP(DBSA) was observed by a JEM-2000EX II transmission electron microscope operated at 100 $\mathrm{kV}$. The film specimens were microtomed at $-50{ }^{\circ} \mathrm{C}$ using a Reichert Ultracut E low-temperature sectioning system. The ultrathin sections were picked onto the copper grids coated with carbon-supporting films followed by staining by exposure to iodine vapor for $30 \mathrm{~h}$. Iodine is a preferential staining agent for P4VP(DBSA), so the P4VP(DBSA) phase appears dark in the TEM micrographs.

\section{Results and Discussion}

Figure 2 shows the SAXS profiles in the high- $q$ region of PS12K- $b$-P4VP12K(DBSA) to demonstrate the formation of the 


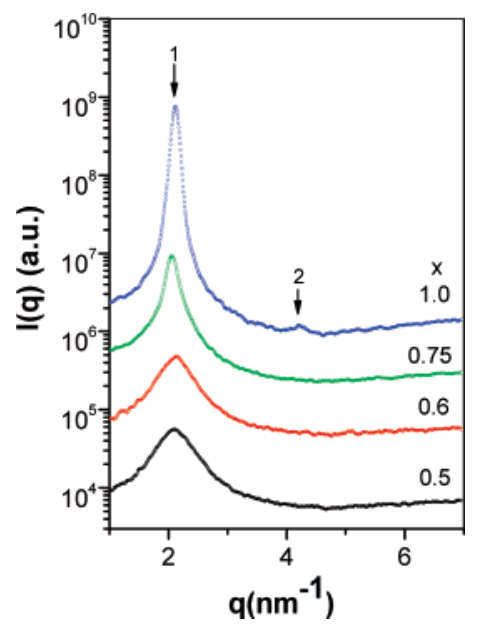

Figure 2. SAXS profiles in the high- $q$ region of PS12K- $b$-P4VP12K(DBSA) comb-coil copolymers with different $x$. The formation of the lamellar mesophase organized by the comb blocks is demonstrated by the presence of a primary peak situating at $2.1 \mathrm{~nm}^{-1}$.

smaller-scale lamellar mesophase organized by the comb blocks. The system with stoichiometric binding $(x=1.0)$ shows a scattering peak at $2.1 \mathrm{~nm}^{-1}$ along with a weak second-order peak, indicating the formation of a lamellar structure with the interlamellar distance of $3.0 \mathrm{~nm}$. The primary scattering peak becomes broader at lower $x$ due to the poorer coherent order of the lamellar stacks. PS50K- $b$-P4VP50K(DBSA) is found to display the same scattering features. The formation of the lamellar mesophase as the matrix phase is also evidenced by the mesomorphic birefrigerent pattern observable under polarized optical microscopy (cf. Supporting Information).

Figure 3a shows the TEM micrograph of PS50K- $b$-P4VP50K(DBSA) with $x=1.0$ for resolving the PS domain structure. The dark matrix corresponds to the P4VP(DBSA) phase preferentially stained by iodine. The formation of cylindrical microdomains by PS blocks is evidenced by the observations of both the images corresponding to the side view (the striation) and the top view (the cross section) of the cylinders in the micrographs with lower magnification. From the spatial arrangement of the cross sections and the six-spot pattern in the corresponding Fourier transform (FT) image in Figure 3a, the PS cylinders are seen to pack in the typical hexagonal lattice. Such a hexagonal packing is also found for the copolymer with $x=0.75$.

Figure $3 b$ displays the representative TEM micrograph of PS50K- $b$-P4VP50K(DBSA) with a lower overall binding fraction of $x=0.5$. Interestingly, the cross-section image and the 4-fold symmetry in the corresponding FT image show that the PS cylinders organize into a tetragonal lattice rather than the typical hexagonal lattice. Tetragonal packing is also observed for the composition of $x=0.6$. The TEM micrographs of PS12K- $b$-P4VP12K(DBSA) demonstrate identical morphological features, namely, the PS cylinders pack in hexagonal and tetragonal lattice at $x=0.75$ and 1.0 and $x=0.5$ and 0.6 , respectively (cf. Supporting Information). This implies that the lattice structure is governed by $x$ but not by the molecular weight of the copolymer.

SAXS profiles in the low- $q$ region have also been collected to establish the packing symmetries. Figure 4 shows the low- $q$ SAXS profiles of the isotropic samples of PS12K- $b$-P4VP12K(DBSA). For $x=1.0$ the SAXS intensity displays a series of lattice peaks with the position ratio of $1: 3^{1 / 2}: 4^{1 / 2}$, signaling the formation of hexagonally packed PS cylinders ${ }^{23}$ with the characteristic domain spacing (D) of $31.4 \mathrm{~nm}$ calculated from

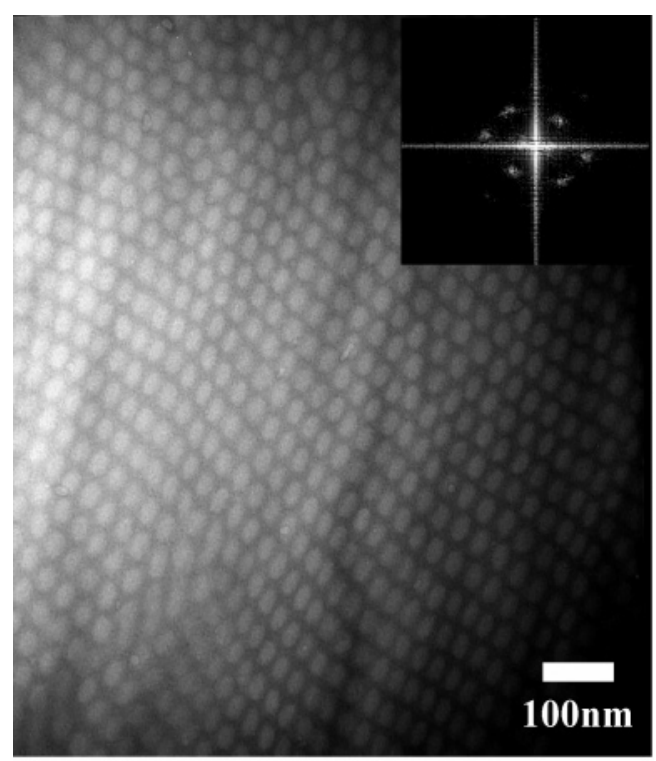

(a)

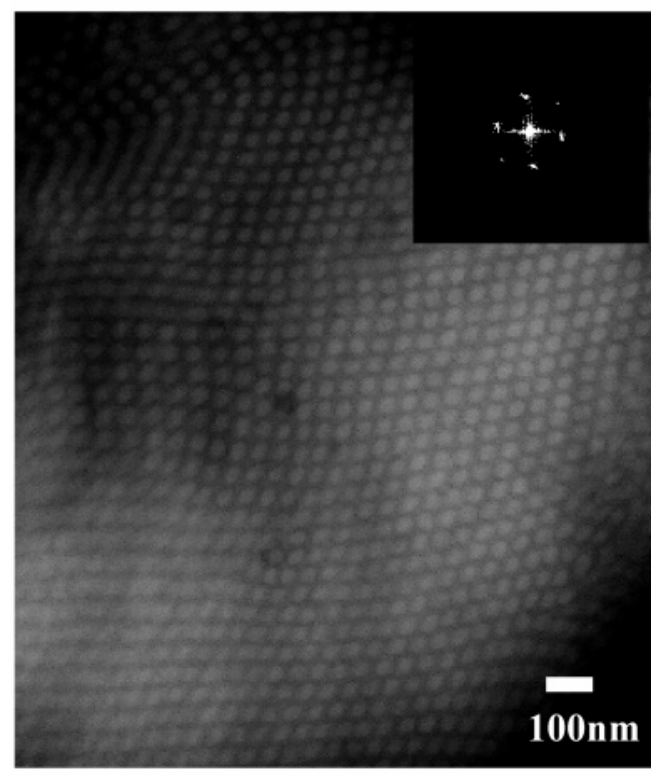

(b)

Figure 3. TEM micrographs showing the cross-sectional view of the cylinders in PS50K- $b$-P4VP50K(DBSA) with (a) $x=1.0$ and (b) $x=$ 0.5 . The Fourier transform patterns of the real-space images are displayed in the upper right corner. The PS cylinders are seen to pack in hexagonal and tetragonal lattice at $x=1.0$ and 0.5 , respectively.

the primary peak position $\left(q_{\mathrm{m}}\right)$ via $D=2 \pi / q_{\mathrm{m}}$. The broad peak marked by " $i=1$ " corresponds to the first maximum of the cylinder form factor. The average radius of the cylindrical domains $(R)$ calculated from the position of this peak $\left(q_{\mathrm{m}}^{i=1}\right)$ via $R=4.98 / q_{\mathrm{m}}{ }^{i=1}$ is $7.8 \mathrm{~nm} .^{24}$

The copolymer with $x=0.5$ exhibits a different scattering pattern. Here a scattering peak situating at ca. $0.4 \mathrm{~nm}^{-1}$ emerges as a shoulder of the primary peak prescribing the domain spacing of $25.5 \mathrm{~nm}$. The positions of the peaks now follow the ratio $1: 2^{1 / 2}: 4^{1 / 2}: 5^{1 / 2}$. The $2^{1 / 2}$ and $5^{1 / 2}$ peaks are not consistent with any diffraction order from the hexagonal lattice; a tetragonal or square lattice can however give rise to these diffraction peaks arising from the (11) and (21) planes. $^{23}$ The radius of the cylinder deduced from the position of the first form factor maximum is $6.5 \mathrm{~nm}$. The scattering pattern remains unperturbed 


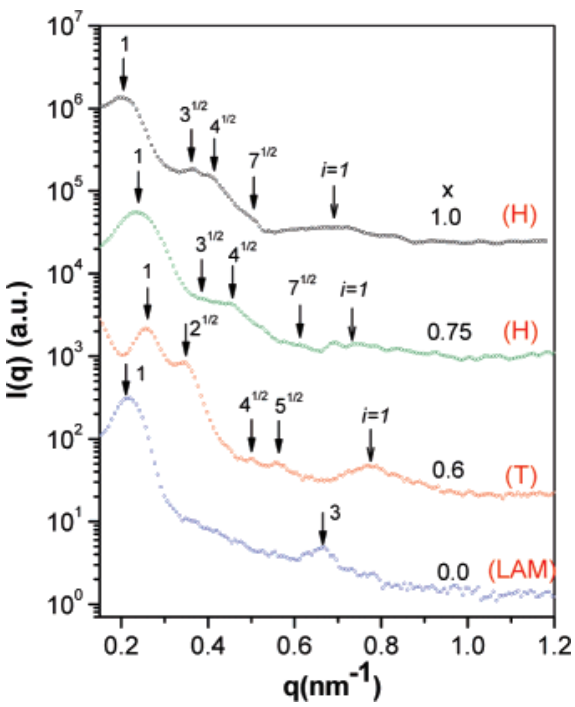

Figure 4. SAXS profiles in the low- $q$ region of PS12K- $b$-P4VP12K(DBSA) comb-coil copolymers with different $x$. The relative positions of the lattice peaks are marked by the arrows. The broad peak marked by " $i=1$ " corresponds to the first maximum of the cylinder form factor. The neat copolymer (i.e., $x=0.0$ ) displays a lamellar morphology due to symmetric composition.

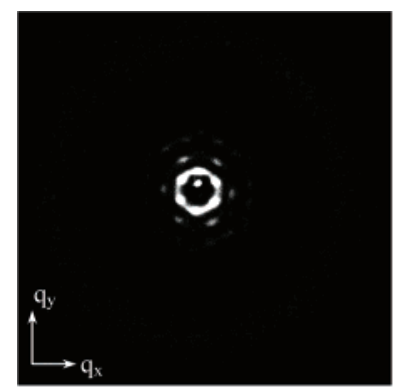

Through view

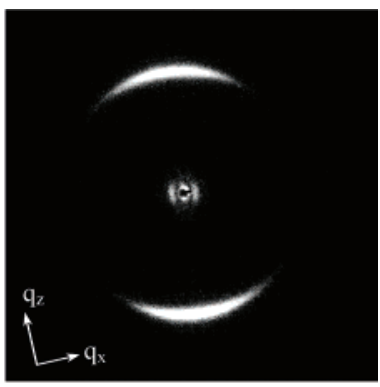

Edge view (a)

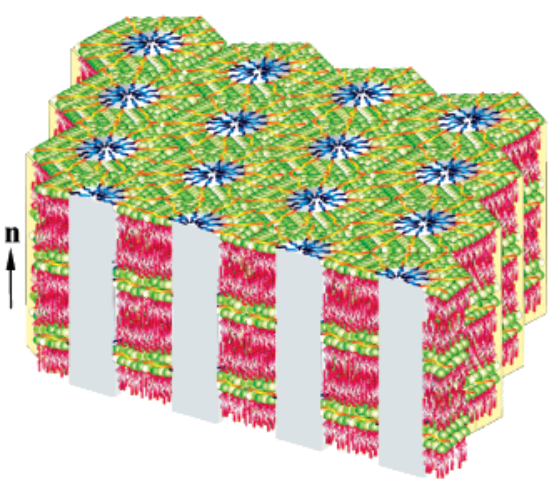

(b)

Figure 5. (a) 2-D SAXS patterns obtained from the through view and the edge view of the shear-aligned PS12K- $b$-P4VP12K(DBSA) combcoil copolymer with $x=1.0$. (b) Schematic illustration of the cylinderwithin-lamellae structure formed at $x=1.0$. The lamellar interface lies perpendicularly to the cylinder axis, yielding the orthogonal orientation.

upon heating to $200{ }^{\circ} \mathrm{C}$, indicating that the tetragonal lattice is thermally stable (cf. Supporting Information).

Two-dimensional (2-D) SAXS patterns are further obtained using the samples with large-scale alignment of the microdomains produced by large-amplitude oscillating shear. The "through-view" and the "edge-view" pattern are obtained by the incident X-ray traveling parallel and perpendicular to the shear flow direction (i.e., $z$-axis), respectively. Figure 5a shows

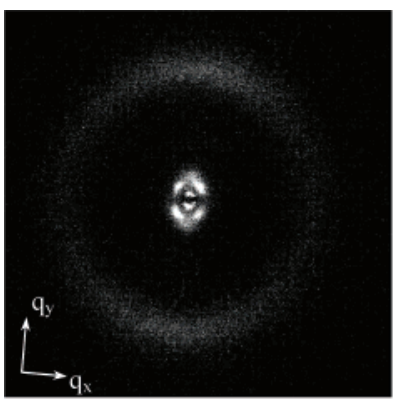

Through view

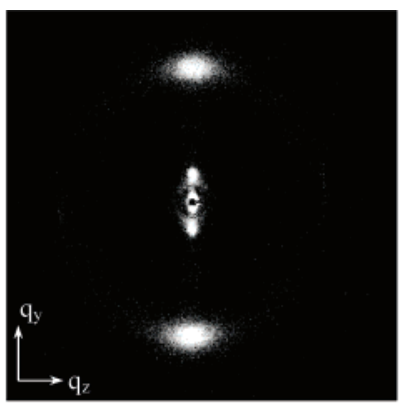

Edge view (a)

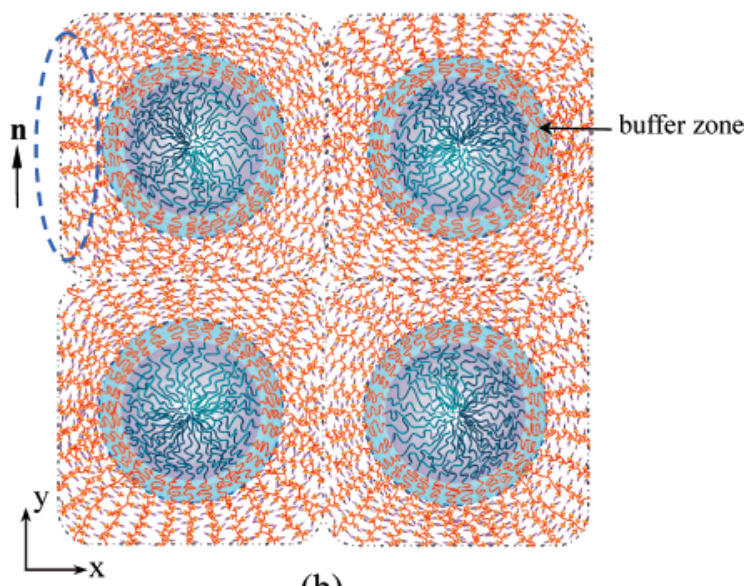

(b)

Figure 6. (a) 2-D SAXS patterns obtained from the through view and the edge view of the shear-aligned PS12K- $b$-P4VP12K(DBSA) combcoil copolymer with $x=0.5$. (b) Schematic illustration proposing the structure formed at $x=0.5$. The DBSA side chains on the comb blocks are proposed to lie flatly on the plane of the cylinder cross section (i.e., the $x y$ plane). A buffer zone is proposed to be formed by the free $4 \mathrm{VP}$ segments in the vicinity of the cylinder interface to alleviate the crowding of the side chains. The normals of the lamellae (n) attached to the lateral sides of the cylinders (e.g., those enclosed by the dashed region) orient relatively perpendicular to the cylinder axis, thereby giving rise to two diffraction spots in the edge-view scattering pattern.

the 2-D SAXS patterns of the system with $x=1.0$. The throughview pattern displays six spots from the (10) planes, indicating that the PS cylinders are aligned in the flow direction and pack with hexagonal symmetry in the plane perpendicular to the flow direction. The edge-view pattern exhibits two arcs at the equator arising from the (10) planes. Two additional arcs associated with the lamellar stacks formed by the comb blocks are identified in the outer region of this 2-D pattern. The fact that these two arcs are located in the meridian indicates that the lamellar interface lies perpendicularly to the cylinder axis, giving rise to the orthogonal orientation as illustrated in Figure 5b. ${ }^{18,25}$

Figure 6a displays the 2-D SAXS patterns of the copolymer with $x=0.5$. Tetragonal packing of the PS cylinders is clearly evidenced by the through-view pattern showing four diffraction spots from the (10) planes in the four quadrants. The smallerscale lamellar structure gives rise to a diffuse ring in the throughview pattern and two spots in the meridian of the edge-view pattern. Most interestingly, the (10) reflections from the PS cylinders in the edge-view pattern are also located in the meridian, which means that the cylinders and the P4VP(DBSA) lamellae do not display the orthogonal orientation found for $x$ $=1.0$. We propose that the DBSA side chains on the comb blocks lie flatly on the plane of the cylinder cross section (i.e., the $x y$ plane), leading to lamellar stacking (and hence an electron density modulation) around the cylinders in the through view, 


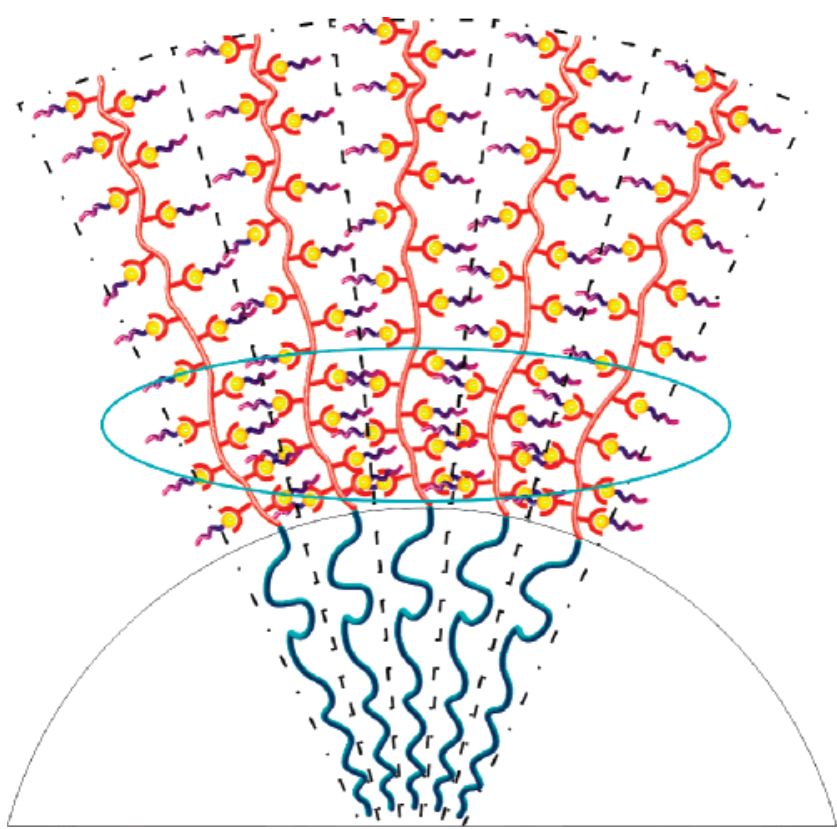

Figure 7. Schematic illustration showing that the side chains (e.g., those bound by the blue) on the comb blocks with high binding density are overly crowded near the cylinder interface if they lie flatly on the $x y$ plane. Such a crowding can be avoided if the side chains align parallel to the cylinder axis, leading to the orthogonal orientation.

as schematically illustrated in Figure $6 \mathrm{~b}$. Such a peculiar edgeon lamellar organization yields a ring in the through-view scattering pattern. In the edge view the normals of the lamellae attached to the lateral sides of the cylinders (i.e., those enclosed by the dashed region in Figure 6b) orient relatively perpendicular to the cylinder axis, and hence results in two spots in the meridian of the corresponding SAXS pattern.

The SAXS result reveals an interesting connection between the packing lattice and the orientation of the smaller-scale lamellae with respect to the larger-scale cylinder. The system showing orthogonal orientation of the two structural units exhibits hexagonal lattice, whereas the edge-on lamellar organization depicted in Figure $6 \mathrm{~b}$ leads to tetragonal packing. The orthogonal orientation, having been observed for other similar systems, ${ }^{16,18,25}$ is favored in that it allows the lamellae to stack regularly over a long distance and hence lowers the free energy of interlamellar interaction. Moreover, considering that the width of the comb block projected onto the $x y$ plane decreases as we approach the cylinder interface along the radial direction, if the comb blocks with high binding density were to lie flatly, the side chains near the cylinder interface would have been overly crowded once those away from the interface attain the normal density, as illustrated in Figure 7 . Such a crowding can be avoided if the side chains align parallel to the cylinder axis, leading to the orthogonal orientation.

At $x=0.5$ and 0.6 where a significant fraction of $4 \mathrm{VP}$ units remain unbound with DBSA, the subchains composing of these units may locate in the vicinity of the cylinder interface, thereby creating a buffer zone to alleviate the crowding of the side chains once they lie flatly (Figure 6b). As a result, the edge-on lamellar arrangement becomes accessible. Outside the buffer zone the tendency of the side chains to attain a uniform degree of stretching for regular lamellar stacking around the cylinder creates rather planar boundaries for the corona (Figure 6b). Cylindrical micelles with such an anomalous boundary then pack into a tetragonal lattice to minimize the corona deformation for space filling. It should be noted that the binding mode of DBSA to P4VP blocks illustrated in Figure $6 \mathrm{~b}$ is analogous to the cooperative binding observed in the complexes of polyelectrolytes with oppositely charged surfactants formed in aqueous media. ${ }^{26-28}$

Nevertheless, the orthogonal orientation is still the favorable structure once the interlamellar interaction governs the lamellar organization. This is the case at high binding densities as the interlamellar interaction is expected to be strong (to the first order of approximation, the interlamellar interaction may be assumed to be proportional to the binding fraction of the comb blocks). The edge-on organization observed at $x=0.5$ and 0.6 must hence be induced by another driving force that outweighs the weaker interlamellar interaction so as to force the lamellae to adopt an unfavorable arrangement. We propose that this driving force originates from the conformational entropy of PS blocks. From the position of the first form factor peak, the average radius of the PS cylinders in the hexagonal lattice is found to be $1.3 \mathrm{~nm}$ larger than that associated with the tetragonal lattice. This means that the PS blocks in the former are more stretched and hence possess a lower conformational entropy. In this case, the significant stretching of P4VP(DBSA) blocks normal to the cylinder interface to form orthogonally oriented lamellae forces the covalently linked PS blocks to stretch accordingly. At lower overall binding fractions, the relaxation of PS blocks to gain higher conformational entropy dominates and forces the DBSA side chains to lie flatly on the $x y$ plane; in this case, the width of the comb block prescribes a larger width for the junction point projected onto the $x y$ plane and hence alleviates the stretching of PS blocks.

\section{Conclusions}

We have shown that the hierarchical assembly of a combcoil diblock copolymer system generates hexagonally or tetragonally packed cylinders embedded in the lamellar mesophase organized by the comb blocks. The packing symmetry is determined by the orientation of the lamellae with respect to the cylindrical domains prescribed by the overall grafting density of the comb blocks. Hexagonal lattice is favored for the orthogonal orientation, while tetragonal packing becomes the stable structure when the lamellae adopt the edge-on arrangement. The main difference between the present system and the conventional coil-coil diblock copolymers lies in the molecular order of the matrix phase, where the cylindrical domains here are embedded in a liquid crystalline matrix rather than a disordered liquid phase. The exploitation of liquid crystallinity is an effective approach for expanding the morphological window of block copolymers. In particular, various novel microdomain structures, including wavy lamellae, ${ }^{29}$ zigzag lamellae, ${ }^{30}$ and arrowhead domains, ${ }^{29}$ have been created through the modification of interfacial curvature by the liquid crystalline order. The present work along with the previous ones ${ }^{13,14}$ attest that the lattice structure can also be modified by the liquid crystalline order. Comparing with the liquid crystalline rodcoil diblock copolymers, the comb-coil system may exhibit more versatile structure variation due to the adjustable mesomorphic order or liquid crystallinity of the comb blocks by the grafting density. This is demonstrated by the accessibility of both hexagonal and tetragonal lattices in a single PS- $b$-P4VP(DBSA) system with different binding fractions.

Acknowledgment. We thank the financial support from the National Science Council under Contract NSC 94-2216-E-007039. National Synchrotron Radiation Research Center (NSRRC), Taiwan on the support of part of the X-ray scattering experiments under the project ID 2005-3-086-3 is also gratefully acknowledged. 
Supporting Information Available: Text discussing the polarized optical microscopy observations and figures showing POM micrographs, TEM micrographs and the corresponding FT images, and temperature-dependent SAXS profiles. This material is available free of charge via the Internet at http://pubs.acs.org.

\section{References and Notes}

(1) Jones, R. A. L. Soft Condensed Matter; Oxford University Press: Oxford, U.K., 2002.

(2) Hamley, I. W. The Physics of Block Copolymers; Oxford University Press: Oxford, U.K., 1998

(3) Leibler, L. Macromolecules 1980, 13, 1602.

(4) Matsen, M. W.; Bates, F. S. J. Chem. Phys. 1997, 106, 2436.

(5) Chen, B.; Baumeister, U.; Pelzl, G.; Das, M. K.; Zeng, X.; Ungar, G.; Tschierske, C. J. Am. Chem. Soc. 2005, 127, 16578.

(6) Cheng, X.; Prehm, M.; Das, M. K.; Kain, J.; Baumeister, U.; Diele, S.; Leine, D.; Blume, A.; Tschierske, C. J. Am. Chem. Soc. 2003, $125,10977$.

(7) Canilho, N.; Kasëmi, E.; Mezzenga, R.; Schlüter, A. D. J. Am. Chem. Soc. 2006, 128, 13998.

(8) Lee, M.; Cho, B.-K.; Oh, N.-K.; Zin, W.-C. Macromolecules 2001, 34, 1987.

(9) Evans, H. M.; Ahmad, A.; Ewert, K.; Pfohl, T.; Martin-Herranz, A.; Bruinsma, R. F.; Safinya, C. R. Phys. Rev. Lett. 2003, 91, 75501.

(10) Liu, Y.-C.; Chen, H.-L.; Su, C.-J.; Lin, H.-K.; Liu, W.-L.; Jeng, U.S. Macromolecules 2005, 38, 9434 .

(11) Mogi, Y.; Nomura, M.; Kotsuji, H.; Ohnishi, K.; Matsushita, Y.; Noda, I. Macromolecules 1994, 27, 6755.

(12) Hückstädt, H.; Göpfert, A.; Abetz, V. Macromol. Chem. Phys. 2000, 201, 296.

(13) Fischer, H. Polymer 1994, 35, 3786.

(14) Tenneti, K. K.; Chen, X.; Li, C. Y.; Tu, Y.; Wan, X.; Zhou, Q.-F.; Sics, I.; Hsiao, B. S. J. Am. Chem. Soc. 2005, 127, 15481.
(15) Ruokolainen, J.; Mäkinen, R.; Torkkeli, M.; Mäkelä, T.; Serimaa, R.; ten Brinke, G.; Ikkala, O. Science 1998, 280, 557.

(16) Ruokolainen, J.; ten Brinke, G.; Ikkala, O. Adv. Mater. 1999, 11, 777.

(17) Ruotsalainen, T.; Torkkeli, M.; Serimaa, R.; Mäkelä, T.; Mäki-Ontto, R.; Ruokolainen, J.; ten Brinke, G.; Ikkala, O. Macromolecules 2003, 36, 9437.

(18) Alberda van Ekenstein, G.; Polushkin, E.; Nijland, H.; Ikkala, O.; ten Brinke, G. Macromolecules 2003, 36, 3684.

(19) Kosonen, H.; Valkama, S.; Ruokolainen, J.; Torkkeli, M.; Serimaa, R.; ten Brinke, G.; Ikkala, O. Eur. Phys. J. E 2003, 10, 69.

(20) Valkama, S.; Kosonen, H.; Ruokolainen, J.; Haatainen, T.; Torkkeli, M.; Serimaa, R.; ten Brinke, G.; Ikkala, O. Nat. Mater. 2004, 3, 872.

(21) Ikkala, O.; ten Brinke, G. Chem. Commun. 2004, 19, 2131.

(22) Bondzic, S.; de Wit, J.; Polushkin, E.; Schouten, A. J.; ten Brinke, G.; Ruokolainen, J.; Ikkala, O.; Dolbnya, I.; Bras, W. Macromolecules 2004, 37, 9517.

(23) Förster, S.; Timmann, A.; Konrad, M.; Schellbach, C.; Meyer, A.; Funari, S. S.; Mulvaney, P.; Knott, R. J. Phys. Chem. B. 2005, 109, 1347.

(24) Tanaka, H.; Hasegawa, H.; Hashimoto, T. Macromolecules 1991, 24 240.

(25) Osuji, C.; Zhang, Y.; Mao, G.; Ober, C. K.; Thomas, E. L. Macromolecules 1999, 32, 7703.

(26) Chandar, P.; Somasundaran, P.; Turro, N. J. Macromolecules 1988, 21,950 .

(27) Bekturov, E. A.; Bimendina, L. A. Adv. Polym. Sci. 1982, 41, 99.

(28) Kabanov, V. A.; Zezin, A. B. Makromol. Chem. Suppl. 1984, 6, 259.

(29) Chen, J. T.; Thomas, E. L.; Ober, C. K.; Mao, G.-P. Science 1996, $273,343$.

(30) Chen, J. T.; Thomas, E. L.; Ober, C. K.; Hwang, S. S. Macromolecules 1995, 28, 1688.

MA0626230 